TITLE:

\title{
Electronic Stress Tensor Study of Aluminum Nanostructures for Hydrogen Storage
}

AUTHOR(S):

Szarek, Pawel; Watanabe, Kouhei; Ichikawa, Kazuhide; Tachibana, Akitomo

\section{CITATION:}

Szarek, Pawel ... [et al]. Electronic Stress Tensor Study of Aluminum Nanostructures for Hydrogen Storage. Materials Science Forum 2010, 638-642: 1137-1142

\section{ISSUE DATE:}

2010-01

URL:

http://hdl.handle.net/2433/124570

\section{RIGHT:}

(c) 2010 Trans Tech Publications; この論文は出版社版でありません。引 用の際には出版社版をご確認ご利用ください。; This is not the published version. Please cite only the published version. 


\title{
Electronic stress tensor study of aluminum nanostructures for hydrogen storage
}

\author{
Pawel Szarek ${ }^{1, a}$, Kouhei Watanabe ${ }^{2, b}, K^{2, d}$ azuide Ichikawa ${ }^{2, c}$ and Akitomo \\ Tachibana ${ }^{2, \mathrm{~d}, *}$ \\ ${ }^{1}$ Wroclaw University of Technology, Institute of Physical and Theoretical Chemistry, Wybrzeze \\ Wyspianskiego 27, 50-370 Wroclaw, Poland \\ 2Department of Micro Engineering, Kyoto University, Yoshidahonmachi, Kyoto, 606-8501, Japan \\ apawel.szarek@pwr.wroc.pl, 'batanabe.kouhei@t03.mbox.media.kyoto-u.ac.jp, \\ 'kazuhide@me.kyoto-u.ac.jp, ${ }^{\text {d, }}{ }^{*}$ akitomo@scl.kyoto-u.ac.jp
}

Keywords: energy density, chemical potential, bond order, metal cluster, hydrogen storage

\begin{abstract}
We report the new structures of aluminum hydrides derived from the $\mathrm{Al}_{4}$ tetrahedral cages. We perform $a b$ initio quantum chemical calculation for these new aluminum hydrides. Our calculation of binding energies of the new aluminum hydrides reveal that stability of these hydrides increases as more hydrogen atoms are adsorbed, while stability of Al-H bonds decreases. We also calculate electronic stress tensor to evaluate the chemical bonds of these hydrides. As a result, we find that the bonds of the $\mathrm{Al}_{4}$ tetrahedral cage are strengthened as more hydrogen atoms are adsorbed on the aluminum hydrides. Our calculation of the potential energy surfaces and the regional chemical potential show that hydrogen atoms are likely to adsorb on bridge site at first.
\end{abstract}

\section{Introduction}

Recently, hydrogen is paid much attention as the new primary energy source because of the depletion of fossil fuels and environmental issues such as global warming. Hydrogen is derived from fossil fuels such as petroleum and natural liquid gas in a present technology. It can be also derived from water if the method is established. Hydrogen is excellent in the point of not generating carbon dioxide that causes global warming at all in the process of combustion and chemical reactions in a fuel cell.

There are three basic research challenges - production, storage, application - for a "hydrogen economy". Our study in this paper concerns hydrogen storage systems. As is emphasized in the recent reports on basic research challenges for hydrogen storage, its high efficiency is a key factor in enabling the success of the hydrogen economy [1,2]. Hydrogen storage systems must exhibit following properties: appropriate thermodynamics, fast kinetics, high storage capacity (more than 10 wt \%), effective heat transfers, high volumetric densities, long cycle lifetime, safety under normal use [3]. To implement these properties, solid-state storage is useful. Metal hydrides, chemical storage materials, nanostructured materials are well known for effective solid-state storage systems. Among them, we investigate aluminum hydrides in the form of aluminum clusters. More specifically, we deal with an aluminum tetrahedral cage $\left(\mathrm{Al}_{4}\right)$ and its hydrides which were recently observed in experiment and confirmed to have enhanced stability [4]. It is also interesting to study the tetrahedral cage in the aspect that it is the fundamental structure of an aluminum icosahedral cage $\left(\mathrm{Al}_{12}\right)$ [5].

\section{Theory and calculation method}

Electronic structure calculation. In this work, optimizations of the geometries of the clusters and electronic structures are calculated by GAUSSIAN03 program package [6] using the Møller-Plesset's second-order perturbation theory (MP2) [7-11] and the DFT with Perdew-Wang 1991 exchange and correlation function (PW91) [12,13]. 6-311G** basis set [14-16] is used for the diffuse and polarization functions. 
Regional DFT calculation. The theoretical method utilized in this study (Regional Density Functional Theory: RDFT) allows one to assign energy density to any point in space according to associated electronic density. The obtained energy density might be decomposed into following energy densities: the kinetic energy density, the external potential energy density, and the interelectron potential energy density [17]. The details of the theory can be found elsewhere [17-23] and here we would like to only briefly review a part of it, directly related to this study. The non-positively defined kinetic energy density $n_{T}(\vec{r})$ [18] plays a particular role in the theoretical method used in this study. The unique concept of electronic drop $\left(R_{\mathrm{D}}\right)$ and electronic atmosphere $\left(R_{\mathrm{A}}\right)$ regions, separated with interface surface $(S)$ is used to define shape of atoms and molecules [18]. In the $R_{\mathrm{D}}$ region, where kinetic energy density $n_{T}(\vec{r})>0$, the classical movement of electrons is granted, while in $R_{\mathrm{A}}\left(n_{T}(\vec{r})<0\right)$ only quantum effects for electrons are possible and the $S$ defines a turning point for an electron. Below, $m$ denotes the electron mass and $v_{i}$ denotes the occupation number of the orbital $\psi_{i}(\vec{r})$. Here,

$$
n_{T}(\vec{r})=-\frac{\hbar^{2}}{4 m} \sum_{i}^{o c c} v_{i}\left[\psi_{\mathrm{i}}^{*}(\vec{r}) \Delta \psi_{\mathrm{i}}(\vec{r})+\Delta \psi_{\mathrm{i}}^{*}(\vec{r}) \psi_{\mathrm{i}}(\vec{r})\right]
$$

and

$$
\begin{array}{ll}
R_{D}: & n_{T}(\vec{r})>0, \\
R_{A}: & n_{T}(\vec{r})<0, \\
S: & n_{T}(\vec{r})=0 .
\end{array}
$$

The electronic structure of system under study is characterized using electronic stress tensor $\tau^{S k l}(\vec{r})[18-23]$

$$
\tau^{S k l}(\vec{r})=\frac{\hbar^{2}}{4 m} \sum_{i}^{o c c} v_{i}\left[\psi_{\mathrm{i}}^{*}(\vec{r}) \frac{\partial^{2} \psi_{\mathrm{i}}(\vec{r})}{\partial x^{k} \partial x^{l}}-\frac{\partial \psi_{\mathrm{i}}^{*}(\vec{r})}{\partial x^{k}} \frac{\partial \psi_{\mathrm{i}}(\vec{r})}{\partial x^{l}}+\frac{\partial^{2} \psi_{\mathrm{i}}^{*}(\vec{r})}{\partial x^{k} \partial x^{l}} \psi_{\mathrm{i}}(\vec{r})-\frac{\partial \psi_{\mathrm{i}}^{*}(\vec{r})}{\partial x^{l}} \frac{\partial \psi_{\mathrm{i}}(\vec{r})}{\partial x^{k}}\right],
$$

which describes the internal distortion of electronic density. The three eigenvalues of stress tensor and their eigenvectors determining principal axis can be used for geometrical visualization of bonding as well as quantitative evaluation of its properties and reactive regions in molecules [24-26]. The trace over the eigenvalues of stress produces energy density $\varepsilon_{\tau}^{S}(\vec{r})$ in non-relativistic limit of the Rigged QED [21],

$$
\varepsilon_{\tau}^{S}(\vec{r})=\frac{1}{2} \sum_{k} \tau^{S k k}(\vec{r}), E=\int d^{3} \vec{r} \varepsilon_{\tau}^{S}(\vec{r})
$$

The total force acting on electrons in the system is composed of Lorentz force $\hat{\vec{L}}^{S}(\vec{r})$ and tension force $\hat{\vec{\tau}}^{s}(\vec{r})$. For system in stationary state the total force at every point in space equals zero, thus Lorentz force exactly cancels the tension force.

$$
\begin{aligned}
& \hat{\overrightarrow{\vec{F}}}^{S}(\vec{r})=\hat{\vec{\tau}}^{S}(\vec{r})+\hat{\vec{L}}^{S}(\vec{r}), \\
& \left\langle\hat{\vec{\tau}}^{S}(\vec{r})\right\rangle+\left\langle\hat{\vec{L}}^{S}(\vec{r})\right\rangle=0,
\end{aligned}
$$




$$
\begin{aligned}
& \tau^{S k}(\vec{r})=\partial_{i} \tau^{S k l}(\vec{r}) \\
& =\frac{\hbar^{2}}{4 m} \sum_{i}^{o c c} v_{i}\left[\psi_{\mathrm{i}}^{*}(\vec{r}) \frac{\partial \Delta \psi_{\mathrm{i}}(\vec{r})}{\partial x^{k}}-\frac{\partial \psi_{\mathrm{i}}^{*}(\vec{r})}{\partial x^{k}} \Delta \psi_{\mathrm{i}}(\vec{r})+\frac{\partial \Delta \psi_{\mathrm{i}}^{*}(\vec{r})}{\partial x^{k}} \psi_{\mathrm{i}}(\vec{r})-\Delta \psi_{\mathrm{i}}^{*}(\vec{r}) \frac{\partial \psi_{\mathrm{i}}(\vec{r})}{\partial x^{k}}\right] .
\end{aligned}
$$

If one investigates the tension force in the bonding regions of molecule in stationary state, one might find a point where (along with condition $\vec{F}^{S}(\vec{r})=0$ ) the tension (as well as Lorentz force) itself will vanish and any force acting on electron density at that point will be zero [18-23]. The point is called a Lagrange point ( $\left.\vec{r}_{\text {Lagrange }}\right)$ and it is a stationary point for electron density distribution in a molecule [24,25]. The properties calculated at $\vec{r}_{\text {Lagrange }}$ can be used to characterize respective interaction between atoms. The energy density based bond order indices were proposed [24,25]:

$$
\begin{aligned}
& b_{\varepsilon}=\frac{\varepsilon_{\tau A B}^{S}\left(\vec{r}_{\text {Lagrange }}\right)}{\varepsilon_{\tau \text { HH }}^{S}\left(\vec{r}_{\text {Lagrange }}\right)}, \\
& b_{\mu}=\frac{\varepsilon_{\tau A B}^{S}\left(\vec{r}_{\text {Lagrange }}\right)}{n_{A B}\left(\vec{r}_{\text {Lagrange }}\right)}\left(\frac{\varepsilon_{\tau H H}^{S}\left(\vec{r}_{\text {Lagrange }}\right)}{n_{H H}\left(\vec{r}_{\text {Lagrange }}\right)}\right)^{-1},
\end{aligned}
$$

where $\varepsilon_{\tau A B}^{S}\left(\vec{r}_{\text {Lagrange }}\right)$ is the energy density at the Lagrange point of bond of interest and $\varepsilon_{\tau H H}^{S}\left(\vec{r}_{\text {Lagrange }}\right)$ is the energy density at Lagrange point of the $\mathrm{H}-\mathrm{H}$ bond in a $\mathrm{H}_{2}$. In $b_{\mu}$ index, $n_{A B}\left(\vec{r}_{\text {Lagrange }}\right)$ and $n_{H H}\left(\vec{r}_{\text {Lagrange }}\right)$ denote corresponding electronic density respectively. The ratio of energy density to electronic density gives a linear approximation of the regional electronic chemical potential $\mu_{R}$ [24-26], thus $b_{\mu}$ index is called chemical potential bond order.

$$
\mu_{R}=\frac{\partial E_{R}}{\partial N_{R}} \approx \frac{\varepsilon_{\tau}^{S}(\vec{r})}{n_{R}(\vec{r})} .
$$

These quantities are calculated by Molecular Regional DFT program (MRDFT) package developed by our group [27].

\section{Results and discussion}

Structures and evaluation of stability by energy. We begin by showing the bare tetrahedral optimized structure of $\mathrm{Al}_{4}$ in Fig. 1 (a). We find that all the six Al-Al bonds have an equal length of $2.74 \mathrm{~A}$ to a great accuracy. $\mathrm{Al}_{4}$ is considered to have a structure very close to a regular tetrahedron. Below, we investigate how hydrogen adsorbs to this structure.

We search stable structures of $\mathrm{Al}_{4} \mathrm{H}_{\mathrm{n}}(\mathrm{n}=1,2,3,4)$ by trying to put $\mathrm{H}$ to every geometrically possible configuration. Fig.1 (b)-(g) show structures of most stable isomer of $\mathrm{Al}_{4} \mathrm{H}_{\mathrm{n}}(\mathrm{n}=2,4,6,8,10$, 12). We search optimized structure of $\mathrm{Al}_{4} \mathrm{H}_{n}(\mathrm{n}=6,8,10,12)$ by adding two hydrogen atoms to the optimized structure of $\mathrm{Al}_{4} \mathrm{H}_{\mathrm{n}-2}$. Although we do not investigate all the geometrically possible configurations, we believe this way is sufficient to find the optimized structures. $\mathrm{Al}_{4} \mathrm{H}_{6}$ has four terminal hydrogen atoms and two bridge hydrogen atoms because all terminal sites of the $\mathrm{Al}_{4}$ cage are already occupied. $\mathrm{Al}_{4} \mathrm{H}_{8}$ has four terminal hydrogen atoms and four bridge hydrogen atoms. In the reaction path from $\mathrm{Al}_{4} \mathrm{H}_{8}$ to $\mathrm{Al}_{4} \mathrm{H}_{10}$, one bond between $\mathrm{Al}-\mathrm{Al}$ is broken and tetrahedral cage is opened up. $\mathrm{Al}_{4} \mathrm{H}_{10}$ has six terminal hydrogen atoms and four bridge hydrogen atoms. There exist sufficient numbers of hands in $\mathrm{Al}_{4} \mathrm{H}_{10}$ to adsorb six terminal hydrogen atoms because of the break of one $\mathrm{Al}-\mathrm{Al}$ bond. $\mathrm{Al}_{4} \mathrm{H}_{12}$ has six terminal hydrogen atoms and four bridge hydrogen atoms and two hollow 
hydrogen atoms. We have tried to adsorb more hydrogen, without success. We could not find a stable structure for $\mathrm{Al}_{4} \mathrm{H}_{14}$. Thus, we believe $\mathrm{Al}_{4} \mathrm{H}_{12}$ is at full saturation.

We plot the total binding energy (B.E.) and the average B.E. of $\mathrm{H}$ atoms for various clusters in Fig.2 (a) and (b). The total B.E. increases as more hydrogen adsorbed. This means adsorption of hydrogen stabilizes the cluster. On the other hand, the average B.E. of $\mathrm{H}$ atoms increases up to $\mathrm{Al}_{4} \mathrm{H}_{6}$ but it decreases at larger clusters than $\mathrm{Al}_{4} \mathrm{H}_{6}$. This is because there increase bridge hydrogen and hollow hydrogen at larger clusters. We point out that larger total B.E. and smaller average B.E. of H atoms are favorable for hydrogen storage systems. This means hydrogen atoms turns into a state such that easily picked up, while clusters become more stable as hydrogen is adsorbed.

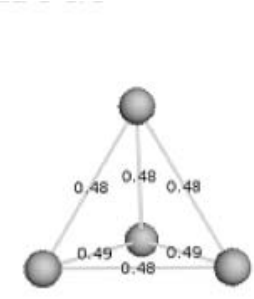

(a) $\mathrm{Al}_{4}$

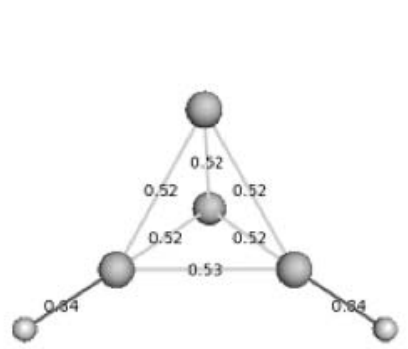

(b) $\mathrm{Al}_{4} \mathrm{H}_{2}$

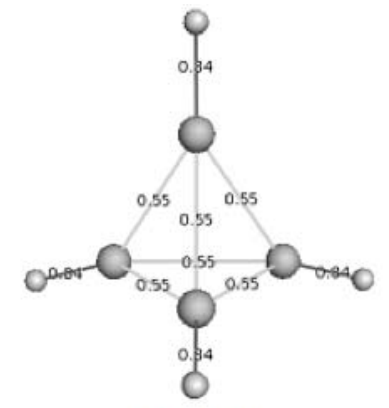

(c) $\mathrm{Al}_{4} \mathrm{H}_{4}$

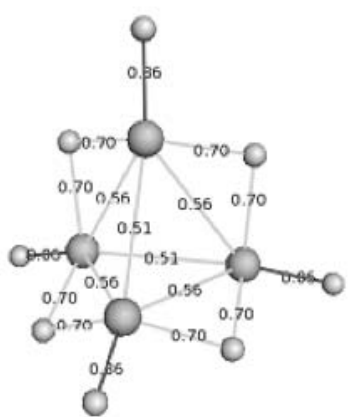

(e) $\mathrm{Al}_{4} \mathrm{H}_{8}$

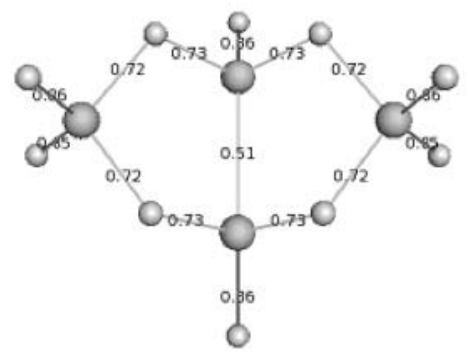

(f) $\mathrm{Al}_{4} \mathrm{H}_{10}$

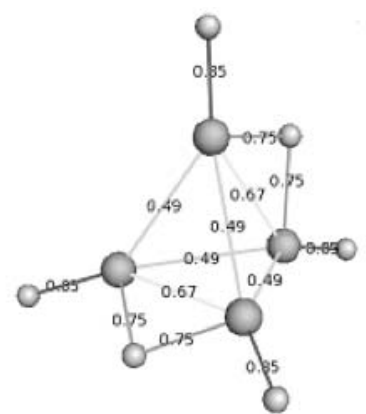

(d) $\mathrm{Al}_{4} \mathrm{H}_{6}$

Fig. 1 Optimized structures of $\mathrm{Al}_{4} \mathrm{H}_{\mathrm{n}}(\mathrm{n}=0,2,4,6,8,10,12)$ and bond orders. The larger balls are $\mathrm{Al}$ and the smaller ones are $\mathbf{H}$.

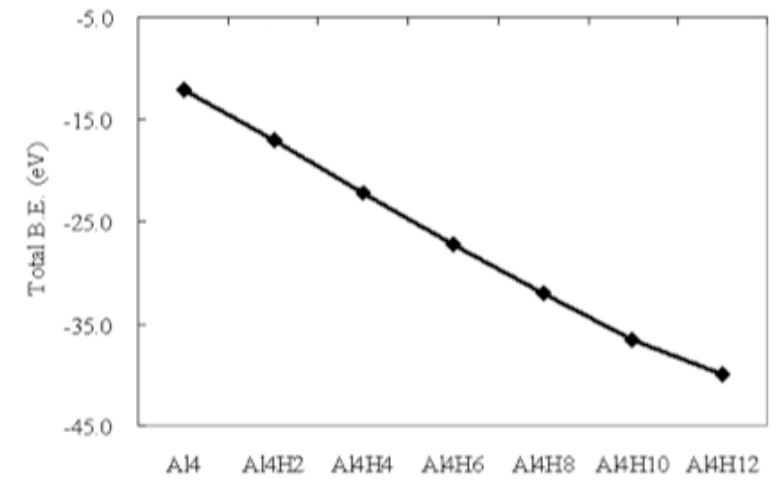

(a) Total binding energies

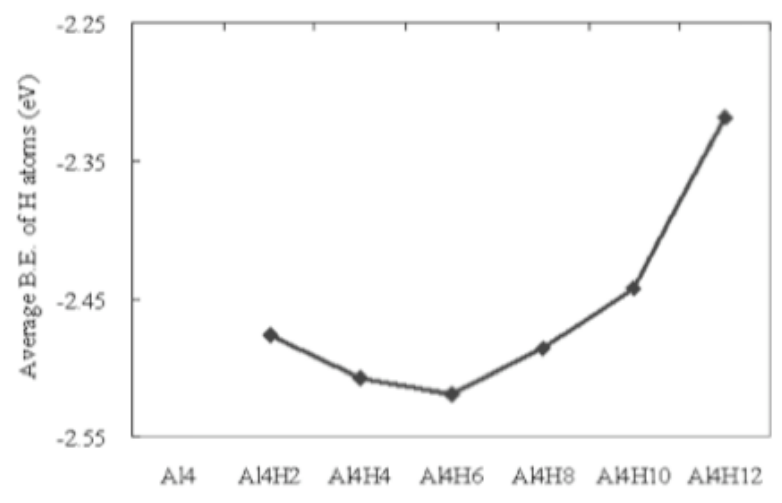

(b) Average binding energies of $\mathrm{H}$ atoms

Fig. 2 Binding energies of $\mathrm{Al}_{4} \mathrm{H}_{n}(\mathrm{n}=0,2,4,6,8,10,12)$.

It is experimentally known that the cluster $\mathrm{Al}_{4} \mathrm{H}_{6}$ exists to be stable [4]. We found the new molecule $\mathrm{Al}_{4} \mathrm{H}_{8}-\mathrm{Al}_{4} \mathrm{H}_{12}$ in this study. It should be noticed that $\mathrm{Al}_{4} \mathrm{H}_{12}$ contains hydrogen by 10.0 $\mathrm{wt} \%$. This high storage capacity exceeds the target value of a hydrogen storage system specified by some technical report [3]. 
Analysis of chemical bond by RDFT. In the previous section, we have found new structures of aluminum hydrides derived from the $\mathrm{Al}_{4}$ tetrahedral cages and have discussed the stability of these molecules by the usual binding energy. In this section, we discuss chemical bonds of these molecules within the RDFT framework introduced above (see the section "Regional DFT calculation").

The chemical potential based bond orders (Eq. (9)) of $\mathrm{Al}_{4} \mathrm{H}_{\mathrm{n}}(\mathrm{n}=0,2,4,6,8,10,12)$ are shown in Fig. 1 by the number on each bond line. We can for example see that addition of hydrogen atoms [(b) $\mathrm{Al}_{4} \mathrm{H}_{2}$ and (c) $\left.\mathrm{Al}_{4} \mathrm{H}_{4}\right]$ strengthens the $\mathrm{Al}-\mathrm{Al}$ bonds. The bond order does not exist between Al's which have a bridge hydrogen between them in (f) $\mathrm{Al}_{4} \mathrm{H}_{10}$ and $(\mathrm{g}) \mathrm{Al}_{4} \mathrm{H}_{12}$. This is because there is no Lagrange point, indicating Al's are not bonded.

Analysis of hydrogen adsorbing process by regional chemical potential. In this section, we discuss the process of hydrogen adsorption on the $\mathrm{Al}_{4}$ tetrahedral cage and its relation to the regional chemical potential defined by Eq. (10). We calculate potential energy curves for $\mathrm{Al}_{4} \mathrm{H}$ when hydrogen atom approaches at three different adsorption sites as functions of distance between $\mathrm{Al}_{4}$ and $\mathrm{H}$. There exist energy barriers in the curves for terminal site and hollow site, while the energy barrier hardly exists in the curve for bridge site. The energy barrier for bridge site adsorption is lowest of the three $(0.0147 \mathrm{eV})$. The energy barriers for terminal site and hollow site adsorption are much larger than that of for bridge site $(0.443 \mathrm{eV}$ for terminal site and $0.416 \mathrm{eV}$ for hollow site, respectively).

When we plot regional chemical potential on the zero surfaces of electronic kinetic energy density which represent the boundary of the molecules, we see that the adsorption point of bridge site has low value, while adsorption point of terminal site and hollow site have relatively high values. Since the region with low chemical potential tends to attract the electron, hydrogen can easily be adsorbed on the point surrounded by the region with low chemical potential. This is considered to be the reason why there exists correspondence between height of activation barrier and that of regional chemicl potential.

\section{Summary}

We have performed $a b$ initio quantum chemical calculation for several clusters of aluminum hydrides derived from the $\mathrm{Al}_{4}$ tetrahedral cages. As a result, we have discovered several aluminum hydrides with new structures like $\mathrm{Al}_{4} \mathrm{H}_{12}$. $\mathrm{Al}_{4} \mathrm{H}_{12}$ have enough mass content $(10.0 \mathrm{wt} \%)$ to be used as hydrogen storage material in real application. We have evaluated and discussed stabilities, chemical bonds and reactions in generation process of these molecules by using various quantities.

We have calculated energies to evaluate the stabilities of these molecules. The total B.E. increases as more hydrogen atoms adsorbed on the molecule, while the average B.E. of $\mathrm{H}$ atoms decreases. Therefore, stability of these molecule increases as more hydrogen atoms are adsorbed, while stability of Al-H bonds decreases.

We have analyzed chemical bonds in these molecules by RDFT method. Hydrogen atoms are adsorbed not by physical adsorption but by covalent bonds in any case. We have quantified that how strength of bonds changes as more hydrogen atoms are adsorbed.

We have studied reactions in generation process of these molecules by calculating energy surfaces and regional chemical potentials. We have found that hydrogen adsorption on the Al-cluster surface is site-dependent. It was found that hydrogen adsorption proceeds without reaction barrier at low chemical potential sites, while at high chemical potential sites there appears an activation barrier.

\section{References}

[1] C. Read, J. Petrovic, G. Ordaz, and S. Satyapal, Mater. Res. Soc. Symp. Proc. 885, A05-01 (2006).

[2] Report by ACIL Tasman and Parsons Brinckerhoff for Australian Government: "National Hydrogen Study," (2003). 
[3] Argonne National Laboratory. Report on Basic Energy Sciences Workshop on Hydrogen Production, Storage, and Use "Basic Research Needs for the Hydrogen Economy," May 13-15, (2003).

[4] A. Grubisic, X. Li, S. T. Stokes, J. Cordes, G. F. Gantefor, K. H. Bowen, B. Kiran, P. Jena, R. Burgert, and H. Schonockel, J. Am. Chem. Soc., 129, 5969 (2007).

[5] A. Goldberg and I. Yarovsky. Phys. Rev. B 75, 195403 (2007).

[6] M. J. Frisch et al, Gaussian 03, Revision B.05, Gaussian, Inc., Pittsburgh PA (2003).

[7] M. Head-Gordon, J. A. Pople, and M. J. Frisch, Chem. Phys. Lett. 153, 503 (1988).

[8] M. J. Frisch, M. Head-Gordon, and J. A. Pople, Chem. Phys. Lett. 166, 275 (1990).

[9] M. J. Frisch, M. Head-Gordon, and J. A. Pople, Chem. Phys. Lett. 166, 275 (1990).

[10]M. Head-Gordon and T. Head-Gordon, Chem. Phys. Lett. 220, 122 (1994).

[11]S. Saebo and J. Almlof, Chem. Phys. Lett. 154, 83 (1989).

[12]J. P. Perdew and Y. Wang, Phys. Rev. B 45, 13244 (1992).

[13]D. R. Hamann, Phys. Rev. B 40, 2980 (1989).

[14]R. C. Binning Jr. and L. A. Curtiss, J. Comp. Chem. 11, 1206 (1990).

[15] L. A. Curtiss, M. P. McGrath, J.-P. Blaudeau, N. E. Davis, R. C. Binning Jr., and L. Radom, J. Chem. Phys. 103, 6104 (1995)

[16] M. P. McGrath and L. Radom, J. Chem. Phys. 94, 511 (1991).

[17]A. Tachibana, Theor. Chem. Acc. 102, 188 (1999).

[18]A. Tachibana, J. Chem. Phys. 115, 3497 (2001).

[19] A. Tachibana, in Stress Induced Phenomena in Metallization, edited by S. P. Baker, (American Institute of Physics, New York, 2002), p. 105.

[20]A. Tachibana, in Reviews in Modern Quantum Chemistry, A Celebration in the Contributions of Robert Parr, edited by K. D. Sen, (World Scientific, Singapore, 2002), Chap. 45, p. 1327.

[21]A. Tachibana, in Fundamental World of Quantum Chemistry, A Tribute to the Memory of Per-Olov Löwdin, edited by E. J. Brändas and E. S. Kryachko, (Kluwer Academic, Dordrecht, 2003), 2, 211.

[22]A. Tachibana, Int. J. Quant. Chem. 100, 981 (2004).

[23]A. Tachibnana, J. Mol. Model. 11, 301 (2005).

[24]P. Szarek and A. Tachibana, J. Mol. Model., 13, 651 (2007).

[25]P. Szarek, Y. Sueda, and A. Tachibana, J. Chem. Phys., 129, 94102 (2008).

[26]P. Szarek, K. Urakami, C. Zhou, H. Cheng, and A. Tachibana, J. Chem. Phys., 130, 084111 (2009).

[27]M. Senami, K. Ichikawa, K. Doi, P. Szarek, K. Nakamura, and A. Tachibana, Molecular Regional DFT program package, ver.3 (Tachibana Lab., Kyoto University, Kyoto, 2008). 